\title{
Providing active antenatal care depends on the place of birth for extremely preterm births: the EPIPAGE 2 cohort study
}

\author{
Caroline Diguisto, ${ }^{1,2,3}$ François Goffinet, ${ }^{1,4}$ Elsa Lorthe, ${ }^{1,5}$ Gilles Kayem, ${ }^{1,5,6}$ \\ Jean-Christophe Roze, ${ }^{7}$ Pascal Boileau, ${ }^{8}$ Babak Khoshnood, ${ }^{1}$ Valérie Benhammou, ${ }^{1}$ \\ Bruno Langer, ${ }^{9}$ Loic Sentilhes, ${ }^{10}$ Damien Subtil ${ }^{11}$ Elie Azria, ${ }^{1,12}$ Monique Kaminski, \\ Pierre-Yves Ancel, ${ }^{1,13}$ Laurence Foix-L'Hélias ${ }^{1,5,14}$
}

- Additional material is published online only. To view please visit the journal online (http://dx.doi.org/10.1136/ archdischild-2016-312322).

For numbered affiliations see end of article.

\section{Correspondence to} Dr Caroline Diguisto, Maternité Olympe de Gouges, Centre Hospitalier, Regional Universitaire Tours, 2 boulevard Tonnellé 37044, France; carolinediguisto@gmail.com

Received 10 November 2016 Revised 29 April 2017 Accepted 2 May 2017 Published Online First 30 June 2017

\section{Linked}

- http://dx.doi.org/10.1136/ fetalneonatal-2017-313332

\section{CrossMark}

To cite: Diguisto C, Goffinet F, Lorthe E, et al. Arch Dis Child Fetal Neonatal Ed 2017:102:F476-F482.

\section{ABSTRACT}

Survival rates of infants born before 25 weeks of gestation are low in France and have not improved over the past decade. Active perinatal care increases these infants' likelihood of survival.

Objective Our aim was to identify factors associated with active antenatal care, which is the first step of proactive perinatal care in extremely preterm births. Methods The population included 1020 singleton births between $22^{0 / 6}$ and $26^{0 / 6}$ weeks of gestation enrolled in the Etude Epidémiologique sur les Petits Ages Gestationnels 2 study, a French national populationbased cohort of very preterm infants born in 2011. The main outcome was 'active antenatal care' defined as the administration of either corticosteroids or magnesium sulfate or delivery by caesarean section for fetal rescue. A multivariable analysis was performed using a two-level multilevel model taking into account the maternity unit of delivery to estimate the adjusted ORs (aORs) of receiving active antenatal care associated with maternal, obstetric and place of birth characteristics.

Results Among the population of extremely preterm births, $42 \%$ received active antenatal care. After standardisation for gestational age, regional rates of active antenatal care varied between 22\% (95\% $\mathrm{Cl} 5 \%$ to $38 \%$ ) and $61 \%(95 \% \mathrm{Cl} 44 \%$ to $78 \%$ ). Despite adjustment for individual and organisational characteristics, active antenatal care varied significantly between maternity units $(p=0.03)$. Rates of active antenatal care increased with gestational age with an aOR of 6.46 (95\% Cl 3.40 to 12.27 ) and 10.09 (95\% Cl 5.26 to 19.36) for infants born at 25 and 26 weeks' gestation compared with those born at 24 weeks. No other individual characteristic was associated with active antenatal care.

Conclusion Even after standardisation for gestational age, active antenatal care in France for extremely preterm births varies widely with place of birth. The dependence of life and death decisions on place of birth raises serious ethical questions.

\section{INTRODUCTION}

In France each year, around 1600 children are born extremely preterm, that is, between 22 and 26 weeks of gestation. ${ }^{1}$ Intensive care increases their survival rate, but they remain at high risk of severe neurodevelopmental sequelae. ${ }^{2}$ Extreme prematurity thus

\section{What is already known on the topic?}

- Survival rates of infants born before 25 weeks are substantially lower in France than in the UK, Sweden, USA, Japan or Australia.

- Active perinatal care increases the chances of survival for extremely preterm infants.

- Decision making for these births is ethically complex due to the high risk of sequelae in survivors.

\section{What this study adds?}

- In France, even after standardisation for gestational age, active antenatal care in cases of extremely preterm births varies widely according to the place of birth.

- In France, even after standardisation for gestational age, active antenatal care in cases of extremely preterm births varies widely according to region of birth.

- In France, active antenatal care in cases of extremely preterm births varies widely according to the maternity unit of birth.

raises serious ethical issues and confronts perinatal teams with a dilemma: whether or not to provide resuscitation at birth. Survival rates of infants born between 22 and 24 weeks of gestation in France have not increased between 1997 and 2011, despite improvements in survival reported in several other countries. ${ }^{13}$ Moreover, the survival rates of infants born before 25 weeks of gestation are substantially lower in France than in Sweden, the USA, Japan or Australia. ${ }^{4-7}$

Active perinatal care increases the chances of both survival and survival without morbidity. ${ }^{8} 9$ Withholding or withdrawing active care may partly explain these low survival rates. The provision of optimal antenatal care is key to the management and survival of extremely preterm infants. Active antenatal care literally means that all available means are used to increase the probability of a live birth so that resuscitation can be performed. Although the factors associated with active care at birth have already been identified, those associated with active 
2484 singleton births between 22 and 26 weeks

included in the EPIPAGE 2 cohort

Termination of pregnancies, stillbirths, livebirths

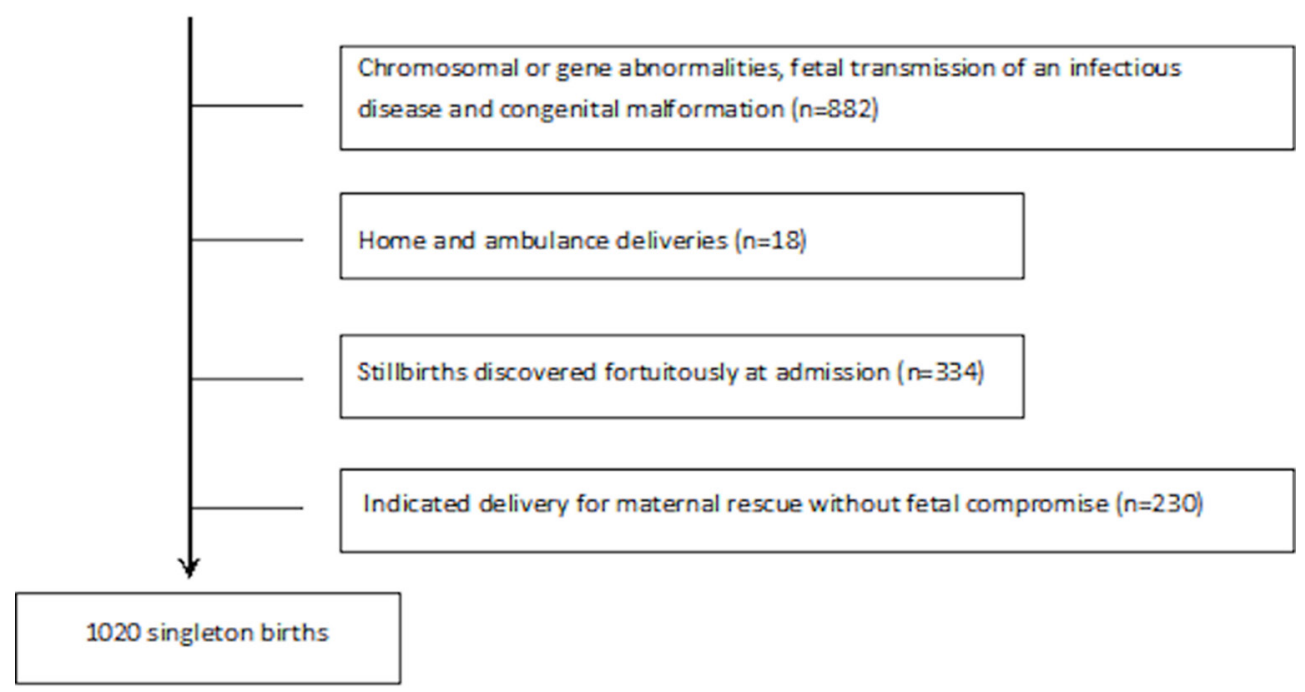

Figure 1 Flow chart of the study population.

antenatal care in this circumstance have never been studied. ${ }^{10-12}$ They might include caregiver or family characteristics or medical or obstetric factors, as well as gestational age, which certainly plays a crucial role. We hypothesised that, regardless of gestational age, the decision to provide active antenatal care depends mostly on place of birth, that is, on local protocols and attitudes to management of extremely preterm births. ${ }^{13}$

The aim of our study was to determine the factors associated with the decision to provide active antenatal care in cases of extremely preterm delivery in France in 2011.

\section{MATERIAL AND METHODS}

\section{Study population}

The study Etude Epidémiologique sur les Petits Ages Gestationnels 2 (EPIPAGE 2) is a population-based cohort study which included all preterm children born between 22 and 34 weeks of gestation (termination of pregnancies, stillbirths and live births) in all the maternity units in 25 French administrative regions (all except one) in $2011 .^{14}$

Families received information and agreed to participate in the study before any data collection began. The appropriate ethics committees, specifically the consultative committee on the treatment of information on personal health data for research purposes (no 10.626) and the committee for the protection of people participating in biomedical research (no CPP SC-2873), approved this study, as did the National Data Protection Authority (CNIL no 911009).

This specific study included all singleton births between $22^{+0}$ and $26^{+6}$ weeks of gestation included in the EPIPAGE 2 cohort for whom obstetricians had the opportunity to provide active antenatal care. Cases involving multiple pregnancies, home or ambulance deliveries and stillbirths discovered fortuitously at admission were excluded; chromosomal or genetic abnormalities, maternal transmission of an infectious disease to the fetus and congenital malformations were also excluded. Births indicated for maternal rescue without fetal compromise, which included caesarean sections for haemorrhage, pre-eclampsia, haemolysis, elevated liver enzymes and low platelets syndrome or other maternal disease were also excluded.

\section{Active antenatal care}

The main outcome, active antenatal care, was defined as administration of corticosteroids (even if the course was not complete) and/or magnesium sulfate for neuroprotection and/or caesarean delivery for fetal rescue. Terminations of pregnancies were never considered active antenatal care, even if corticosteroids or magnesium sulfate were administered or delivery was by caesarean section.

\section{Factors studied}

Individual and organisational (hospital) characteristics were collected prospectively. Maternal individual factors were social, demographic, medical and obstetric characteristics, including the main cause of preterm birth, classified as preterm labour, preterm premature rupture of membranes, hypertensive disorder with or without fetal growth restriction, placental abruption or fetal growth restriction without hypertensive disorders. ${ }^{15}$ Newborn characteristics were sex, birth weight (used as a proxy for antenatal estimation of fetal weight) and gestational age at birth, defined as completed weeks of gestation and based on the last menstrual period and ultrasound assessment. We distinguished three different statuses at birth: terminations of pregnancies, stillbirths and live births. Among live births, we studied admission to the neonatal intensive care unit (NICU) and survival to discharge.

The organisational or maternity unit factors studied were the level of perinatal care and the region of the hospital of birth. Level of perinatal care is defined by official French regulations on the safety of childbirth: level 1 units have no neonatal ward and are not required to have a paediatrician onsite; level 2 units have neonatology facilities to manage infants born at 32 weeks of gestation or later and require the presence of a paediatrician during the day and either their presence or on-call availability at 
Table 1 Characteristics of the study population

$\mathrm{N}=1020, \mathrm{n}(\%)$

\begin{tabular}{|cc|}
\hline $\begin{array}{l}\text { Distribution of women according to the level of perinatal care of maternity unit of } \\
\text { delivery }\end{array}$ & $91(9)$ \\
\hline 1 & $268(26)$ \\
\hline 2 & $661(65)$ \\
\hline 3 & $750(74)$ \\
\hline Momen with a French nationality & $824(81)$ \\
\hline Age of the mother (years)* & $28.9(5.9)$ \\
\hline Multipara & $455(45)$ \\
\hline Regular antenatal care during pregnancy & $837(82)$ \\
\hline Pregnancy after assisted reproductive technology & $84(8)$ \\
\hline Cause of preterm birth & \\
\hline Preterm labour & $512(50)$ \\
\hline Preterm premature rupture of membranes & $351(35)$ \\
\hline Hypertensive disorders without fetal growth restriction & $11(1)$ \\
\hline Hypertensive disorders with fetal growth restriction & $23(2)$ \\
\hline Placental abruption & $11(1)$ \\
\hline Fetal growth restriction without hypertensive disorders & $70(7)$ \\
\hline Gestational age of birth (weeks)* & $24.1(1.4)$ \\
\hline Birth weight in grams & $195(19)$ \\
\hline$<500$ & $181(18)$ \\
\hline $500-600$ & $161(16)$ \\
\hline $600-700$ & $441(43)$ \\
\hline$>700$ & $451(44)$ \\
\hline Female newborn & \\
\hline
\end{tabular}

*Mean (SD).

night and weekends; and level 3 units have an onsite NICU and a neonatologist onsite 24 hours a day, 7 days a week. ${ }^{15}$

\section{Statistical analysis}

The initial descriptive analysis examined the population's baseline characteristics and rates of active antenatal care, status at birth and mortality rates according to gestational age at birth. To ensure that active antenatal care was associated with improved outcomes in our population, we calculated the ORs of live birth and of survival to discharge using two-level hierarchical (women/maternity unit) logistic regression models to adjust for gestational age.

Standardised rates of active antenatal care (percentages with their 95\% CIs) were calculated for each region. As regional rates of active antenatal care may vary with the distribution of gestational age of births within regions, the rates were standardised for gestational age of births. Standardisation was by the overall gestational age distribution of the sample.

Third, we studied factors associated with the provision of active antenatal care with two-level hierarchical (women/ maternity unit) logistic regression models. Our analysis sought to determine whether delivery of active antenatal care varied between maternity units. If it showed a 'maternity unit effect', we sought to assess the extent to which differences in active antenatal care practices between units might be explained by individual or organisational factors. Finally, we sought to identify factors associated with the provision of active antenatal care.

First, an empty model (no variables) enabled us to investigate variations of rates of active antenatal care between maternity units and obtain the baseline maternity-unit level variance (model 0 , variance 0 ). Another model, including gestational age (model 1), allowed us to investigate the association between active antenatal care and gestational age. The next model included all individual variables selected either because they are known to be associated with active antenatal care or because we hypothesised they could possibly be associated with active antenatal care, whether or not these variables were associated with active antenatal care in the univariable analysis (model 2). The final model added level of perinatal care as a predictor variable after adjustment for individual-level variables (model 3). We used the proportional change in the variance (PCV) (defined as $\left.\left(\operatorname{var}^{(\mathrm{n})}-\operatorname{var}^{(0)} / \operatorname{var}^{(0)}\right)\right)$ to assess the extent to which differences between maternity units in their practices of active antenatal care might be explained by the different variables in each model. ${ }^{16}$ The reference variance used to calculate the PCV was (var 0) from model 0. Associations were quantified by adjusted OR (aORs) and their 95\% CIs.

Stata V.13.0 software was used for all analyses.

\section{Missing data}

All variables included in the models had less than $15 \%$ of missing data, and a missing data class was added for variables for which the percentage of missing data was between 5\% and 15\%. Analyses restricted to complete cases and using multiple imputations (chained equations with a logistic regression imputation model for missing binary data and a multinomial imputation model for missing categorical data) were performed to check that the chosen strategy for handling missing data did not induce bias.

\section{RESULTS}

The study population included 1020 singleton births in 244 maternity units in 24 French regions. In one of the 25 regions participating in the EPIPAGE 2 study, no birth met our inclusion criteria (figure 1). The terminations of pregnancies included

Table 2 Active antenatal care, status at birth and mortality rates by gestational age

\begin{tabular}{|c|c|c|c|c|c|c|}
\hline & 22 weeks & 23 weeks & 24 weeks & 25 weeks & 26 weeks & Total \\
\hline & $\mathrm{n}=185$ & $\mathrm{n}=187$ & $\mathrm{n}=188$ & $\mathrm{n}=190$ & $n=270$ & $\mathrm{n}=1020$ \\
\hline Active antenatal management, $\mathrm{n}(\%$ of total) & $4(2.2)$ & $9(4.8)$ & $72(38.3)$ & $134(70.5)$ & $212(78.5)$ & $431(42.2)$ \\
\hline Antenatal steroids, $\mathrm{n}$ (\% of total) & $2(1.1)$ & $8(4.3)$ & $70(37.2)$ & $123(64.7)$ & $193(71.5)$ & $396(38.8)$ \\
\hline Caesarean delivery, $\mathrm{n}$ ( $\%$ of total) & $3(1.6)$ & $2(1.1)$ & $10(5.3)$ & $42(22.1)$ & $114(42.2)$ & $171(16.8)$ \\
\hline Magnesium sulfate, $\mathrm{n}$ ( $\%$ of total) & 0 & 0 & $2(1.1)$ & $8(4.2)$ & $11(4.1)$ & $21(2.1)$ \\
\hline Terminations of pregnancy, $n$ (\% of total) & $26(14.0)$ & $17(9.1)$ & $13(6.9)$ & $14(7.4)$ & $9(3.3)$ & $79(7.7)$ \\
\hline Stillbirths, n (\% of total) & $125(67.6)$ & $118(63.1)$ & $59(31.4)$ & $21(11.0)$ & $23(8.5)$ & $346(33.9)$ \\
\hline Live births, $\mathrm{n}$ (\% of total) & $34(18.4)$ & $52(27.8)$ & $116(61.7)$ & $155(81.6)$ & $238(88.1)$ & $595(58.3)$ \\
\hline Deaths in delivery room, $\mathrm{n}$ ( $\%$ of live births) & $33(97.0)$ & $47(90.0)$ & $52(45.0)$ & $13(8.0)$ & $11(4.0)$ & $156(26.0)$ \\
\hline Deaths in NICU, $\mathrm{n}$ (\% of live births) & $1(3.0)$ & $5(10.0)$ & $31(27.0)$ & $48(31.0)$ & $50(21.0)$ & $135(23.0)$ \\
\hline Alive at discharge, $n$ ( $\%$ of live births) & 0 & 0 & $33(28.0)$ & $94(60.0)$ & $177(74.0)$ & $304(51.0)$ \\
\hline
\end{tabular}

NICU, neonatal intensive care unit 


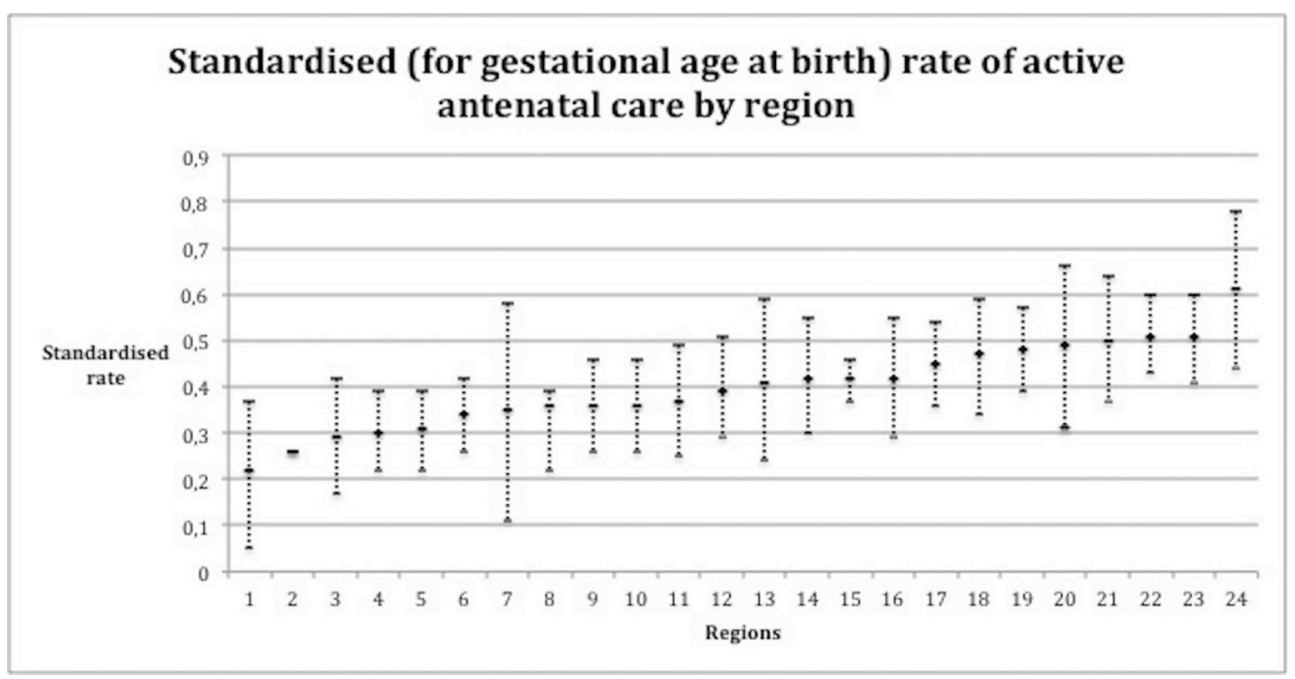

Figure 2 Standardised rates (for gestational age at birth) of active antenatal care and admission to neonatal intensive care unit in 24 French regions.

$(n=79)$ were indicated either for severe growth restriction $(n=29)$ or premature rupture of membranes $(n=34)$ or both $(\mathrm{n}=9)$. Table 1 summarises the study population's baseline characteristics.

Overall $42 \%$ of our population of extremely preterm births received antenatal active management; $58 \%$ of the extremely preterm infants were born alive, and $51 \%$ of the latter survived to discharge. The rates of active antenatal care, live births and survival to discharge increased with gestational age at birth (table 2). Only 2\% (4/185) of the infants born at 22 weeks and $5 \%(9 / 187)$ of those born at 23 weeks received active antenatal care and $18 \%(34 / 185)$ and $28 \%(52 / 187)$, respectively, were born alive. No infant born at 22 or 23 weeks survived to discharge. Among the 188 births at 24 weeks, 38\% (72/188) received active antenatal care: antenatal steroids were administered for $37 \%$, and $5 \%$ of deliveries were caesareans. The rate of live births was 62\% (116/188), and 28\% (33/116) survived to discharge. At 25 and 26 weeks, more than $70 \%$ of the infants received active antenatal care. The live birth rate ranged from $80 \%(155 / 190)$ to $90 \%(238 / 270)$ and the survival to discharge rate from $60 \%(94 / 155)$ to $75 \%$ (177/238) (among live births).

Active antenatal care, after adjustment for gestational age and taking into account the maternity unit of birth, was associated with higher odds of live birth (aOR 12.32, 95\% CI 7.50 to 20.24) and higher odds of survival to discharge for live births (aOR 3.58, 2.15 to 5.98).

Crude rates of active antenatal care in the different regions varied between $21 \%$ and $62 \%$; after standardisation for gestational age at birth, they varied from $22 \%(0.05$ to 0.38$)$ to $61 \%$ (0.44 to 0.78 ) (figure 2).

As seen in table 3, the empty multilevel model (no individual variables) showed a significant variation in active antenatal care between maternity units $(p<0.001)$. The table also includes the calculation of the PCV for each model to assess the extent to which differences in active antenatal care practices might be explained by the different variables: model 1, which had only gestational age as a predictor variable, had a variance of 1.35 with a PCV of 0.12 , that is, $12 \%$ of the residual variance between maternity units could be explained by the differences between them in gestational age at birth. In model 2, adjusted for all individual characteristics, variance decreased to 1.11 and the PCV was 0.27 , that is, $27 \%$ of the residual variance between maternity units could be explained by individual variables. When all individual and organisational variables were included in the model 3, the PCV reached 66\%, that is, $66 \%$ of the difference in the practice of active antenatal care between units could be attributed to these variables. Adjustment for all variables also showed that rates of active antenatal care increased with gestational age at birth, with aORs of 6.46 (3.40 to 12.27$)$ and 10.09 (5.26 to 19.36$)$ for infants born at 25 and 26 weeks in comparison with those born at 24 weeks. The odds of receiving active antenatal care were approximately fourfold lower in level 1 (aOR 0.23 , 95\% CI 0.09 to 0.58 ) and level 2 (aOR $0.2195 \%$ CI 0.11 to 0.37 ) maternity units compared with level three units. Variations of active antenatal care between maternity units were still significant after adjustment for all variables $(p=0.03)$.

Results of this analysis were compared with those of the complete case analyses and analyses with multiple imputations (see online Supplementary appendix). Based on this comparison, we found that our strategy for handling missing data did not induce any bias for estimating the effects of factors associated with active antenatal care.

\section{DISCUSSION}

The results of our study suggest that extremely preterm births in France are managed differently not only according to gestational age but also according to the place of delivery. This finding indicates that local protocols and attitudes to management vary between maternity units. Despite standardisation for gestational age, rates of active antenatal care varied dramatically between regions. Moreover, after we took into account the differences between maternity units and between women attending them (mothers' individual characteristics), differences in the provision of antenatal care for extremely preterm births remained. Moreover, active antenatal care increased the chances of live birth and of survival to discharge for these extremely preterm births.

Strengths of our study include the prospective enrolment of patients and the prospective collection of data (questionnaires specifically designed to study perinatal interventions for preterm births). With one of the largest cohorts of preterm births in Europe, we had sufficient power and precision for estimations. This national population-based study provided a unique 


\section{Original article}

Table 3 Association between active antenatal care and individual or organisational characteristics using two-level (women/maternity unit) models

\begin{tabular}{|c|c|c|c|c|}
\hline & Model 0 & Model 1 & Model 2 & Model 3 \\
\hline \multicolumn{5}{|l|}{ Level of perinatal care of maternity unit of delivery } \\
\hline 1 & & & & 0.23 (0.09 to 0.58$)$ \\
\hline 3 & & & & 1 \\
\hline \multicolumn{5}{|l|}{ French nationality } \\
\hline Yes & & & 1 & 1 \\
\hline \multicolumn{5}{|l|}{ Marital status } \\
\hline Single & & & 0.52 (0.26 to 1.06$)$ & 0.59 (0.29 to 1.17$)$ \\
\hline Married or cohabiting & & & 1 & 1 \\
\hline Missing & & & $0.36(0.12$ to 1.09$)$ & 0.52 (0.17 to 1.56$)$ \\
\hline \multicolumn{5}{|l|}{ Age } \\
\hline No & & & 1 & 1 \\
\hline Yes & & & 0.41 (0.06 to 2.76 ) & 0.36 (0.06 to 2.20$)$ \\
\hline \multicolumn{5}{|l|}{ Parity } \\
\hline Multipara & & & 1 & 1 \\
\hline Primipara & & & $0.97(0.61$ to 1.53$)$ & 0.99 (0.64 to 1.56$)$ \\
\hline \multicolumn{5}{|l|}{ Infertility treatment } \\
\hline Yes & & & 1.02 (0.43 to 2.43$)$ & 1.07 (0.45 to 2.46$)$ \\
\hline No & & & 1 & 1 \\
\hline \multicolumn{5}{|l|}{ Regular consultation during pregnancy } \\
\hline Yes & & & 1 & 1 \\
\hline Fetal growth restriction without hypertensive disorders & & & 0.13 (0.04 to 0.44$)$ & 0.13 (0.04 to 0.44$)$ \\
\hline \multicolumn{5}{|l|}{ Gestational age in weeks } \\
\hline 22 & & 0.03 (0.01 to 0.08$)$ & $0.04(0.01$ to 0.16$)$ & $0.06(0.02$ to 0.20$)$ \\
\hline 23 & & 0.06 (0.03 to 0.14$)$ & 0.07 (0.03 to 0.18$)$ & 0.09 (0.04 to 0.23$)$ \\
\hline 24 & & 1 & 1 & 1 \\
\hline 25 & & 4.63 (2.77 to 7.76$)$ & 7.19 (3.73 to 13.86$)$ & $6.46(3.40$ to 12.27$)$ \\
\hline 26 & & 7.52 (4.55 to 12.43$)$ & 11.71 (5.97 to 22.99$)$ & 10.09 (5.26 to 19.36$)$ \\
\hline \multicolumn{5}{|l|}{ Birth weight } \\
\hline$<500$ & & & 1 & 1 \\
\hline $500-600$ & & & $5.30(1.58$ to 17.73$)$ & 5.16 (1.56 to 16.99$)$ \\
\hline $600-700$ & & & $4.46(1.28$ to 15.62$)$ & 3.84 (1.11 to 13.26$)$ \\
\hline$>700$ & & & $3.58(0.99$ to 12.97$)$ & 3.30 (0.92 to 11.82$)$ \\
\hline \multicolumn{5}{|l|}{ Sex } \\
\hline Girl & & & 0.91 (0.59 to 1.40$)$ & 0.86 (0.56 to 1.32 ) \\
\hline Boy & & & 1 & 1 \\
\hline Variance at the maternity unit level & 1.53 & 1.35 & 1.11 & 0.52 \\
\hline Test of the residual variance & $<0.001$ & $<0.001$ & $<0.001$ & 0.03 \\
\hline Proportional change of the variance & & 0.12 & 0.27 & 0.66 \\
\hline
\end{tabular}


opportunity to study factors associated with active antenatal care of extremely preterm births.

In the absence of a consensual definition of active antenatal care, we reviewed the existing definitions. ${ }^{9}$ Guinsburg et al considered that antenatal care was active when corticosteroids were administered or a caesarean delivery was performed. ${ }^{17}$ Serenius et al added to the definition of active care delivery in a level 3 maternity unit and tocolytic treatment. ${ }^{18}$ Tocolysis was not included as it was considered inappropriate for non-spontaneous preterm births. Its administration, like antenatal transfer, does not necessarily imply that the physician's aim is to provide resuscitation at birth. ${ }^{19}$

A study comparing obstetrical interventions in 10 regions of Europe previously showed geographic variations of obstetric practices in cases of extremely preterm births, but its analysis did not take any individual or organisational factors into account. ${ }^{9}$ Rates of antenatal steroids, antenatal transfer and caesarean section for fetal reasons varied significantly by gestational age and region. At 22 and 23 weeks' gestation, these interventions took place least often in France and in the Netherlands, and most frequently in Germany, Poland and Portugal. That study's finding that obstetric interventions were associated with lower mortality is concordant with ours that active antenatal care is associated with a higher chance of live birth and of survival to discharge. The use of our multilevel model allowed us to take into account the maternity unit of birth and numerous individual medical and obstetric variables that might influence physicians' attitudes. We have shown differences of antenatal practice between regions and between units but failed to find a model which would take into account the maternity unit and the region of birth (a three-level model looking at both maternity unit and regional variations did not converge). Variation for antenatal care between regions may be due to the maternity unit effect but we could not test this hypothesis with our data.

Obstetricians and midwives are often the first medical contact for women at risk of extremely preterm delivery. The information given to parents will depend on gestational age but also on local guidelines and experience and, probably, the individual beliefs of the healthcare providers. Some national guidelines are precise and explicit, such as those in the Netherlands, which advise proactive care from $24^{0 / 6}$ onwards, antenatal transfer from $23^{4 / 6}$ and corticosteroid administration from $23^{5 / 6} \cdot{ }^{20}$ Other national guidelines, such as those in France, are not as explicit and often define a 'grey zone' that varies between countries and advises physicians to provide care according to the parents' preference and what they consider to be the 'best interests of the child'. The information that parents receive will therefore depend on the physician's knowledge of survival and morbidity risks.

The low survival rate of extremely preterm infants in France is cause for concern, especially after the recent report of the Extreme Preterm Infants in Sweden Study (EXPRESS) cohort results suggesting that active perinatal care reduces mortality in this population without increasing neurodevelopmental morbidity. ${ }^{21}$ The EPIPAGE 1 cohort had showed no survival for infants born at 22 or 23 weeks in 1997, and recommendations issued in 2010 by the French Paediatric Society for extremely preterm births defined a grey zone in which decisions should be taken on a case-by-case basis with the parents. ${ }^{22-24}$ Transmission to parents of pessimistic information about the high risk of sequelae and maternal morbidity in caesarean deliveries at these gestational ages may promote the more frequent demand for palliative care.
Another worrisome issue is the significant heterogeneity in the rates of initiation of active antenatal care for extremely preterm newborns across maternity units and regions. It suggests that the chance of survival for extremely preterm infants are not the same everywhere in France and may depend on local protocols and/or regional policies. This situation impairs the cornerstone of equality, one of the three founding principles of the French constitution and a pillar of the United Nation's convention on the rights of children, which states that every child has "the right ... to the enjoyment of the highest attainable standard of health". 25

A national discussion is thus needed to address these points and to propose national guidelines as in other countries. ${ }^{26}$ Such guidelines should deal with both the information provided to parents about and the management of extremely preterm infants. It might at the same time increase the rate of initiation of active care for extremely preterm children and reduce the geographic inequalities of survival.

\section{CONCLUSION}

The low survival rates of extremely preterm infants in France can be partly explained by differences in the practices of active antenatal care between maternity units.

\section{Author affiliations}

${ }^{1}$ Inserm UMR 1153, Obstetrical, Perinatal and Pediatric Epidemiology Research Team (Epopé), Center for Epidemiology and Statistics Sorbonne Paris Cité, DHU Risks in

Pregnancy, Paris Descartes University, Paris, France

${ }^{2}$ Maternité Olympe de Gouges, Centre Hospitalier Regional Universitaire Tours, Tours, France

${ }^{3}$ Université François Rabelais, Tours, France

${ }^{4}$ DHU Risk in Pregnancy, Maternité Port Royal Paris Descartes University Cochin Broca Hotel Dieu Hospitals Assistance publique des hopitaux de Paris, Paris, France ${ }^{5}$ UPMC Univ Paris 06, Sorbonne Universités, Paris, France

${ }^{6}$ Service de Gynécologie Obstétrique, Paris, France

${ }^{7}$ Service de Néonatologie, CIC 004, INSERM, Nantes University Hospital, Nantes, France

${ }^{8}$ Service de Néonatologie, CHI Poissy St-Germain-en-Laye, University Versailles

StQuentin-en-Yvelines, Versailles, France

${ }^{9}$ Pole de Gynécologie Obstétrique, Hôpital de Hautepierre, Strasbourg, France

${ }^{10}$ Department of Obstetrics and Gynecology, University Hospital Bordeaux, Bordeaux, France

${ }^{11}$ Hôpital Jeanne de Flandre, CHRU-University, Lille Nord, France

${ }^{12}$ Maternité Notre Dame de Bon Secours, Groupe Hospitalier Paris Saint Joseph, ParisDescartes University, DHU Risk in Pregnancy, Paris, France

${ }^{13}$ URC CIC P1419, DHU Risk in Pregnancy, Cochin Hotel Dieu Hopital APHP, Paris, France

${ }^{14}$ Service de Néonatologie, Hopital Armand Trousseau, APHP, Paris, France

Acknowledgements The authors are grateful for the participation of all the families of extremely preterm infants in the EPIPAGE-2 cohort study; grateful to all the participating maternity units and also for those who contributed to the study in particular the Obstetric and Extreme Prematurity EPIPAGE-2 working groups.

Contributors CD, FG, GK, PYA, EA and LFH conceived and designed the study. VB participated in the data collection. CD, FG, LFH and BK performed the statistical analysis. CD, FG and LFH interpreted the data helped by EL, GK, JCR, PB, MK and PYA. CD, FG, LFH, PYA and EA wrote the manuscript. EL, GK, JCR, PB, BL, LS, DS and MK reviewed the manuscript and made significant changes. All authors read and approved the final manuscript.

Competing interests None declared.

Patient consent Consent obtained from the guardian.

Ethics approval Consultative Committee on the Treatment of Information on Personal Health Data for ResearchPurposes (no 10.626); Committee for the Protection of People participating in Biomedical Research (no CPP SC-2873); The National Data Protection Authority(CNIL no 911009).

Provenance and peer review Not commissioned; externally peer reviewed.

(c) Article author(s) (or their employer(s) unless otherwise stated in the text of the article) 2017. All rights reserved. No commercial use is permitted unless otherwise expressly granted. 


\section{REFERENCES}

1 Ancel PY, Goffinet F, Kuhn P, et al. Survival and morbidity of preterm children born at 22 through 34 weeks' gestation in France in 2011: results of the EPIPAGE-2 cohort study. JAMA Pediatr 2015;169:230-8.

2 Moore GP, Lemyre B, Barrowman N, et al. Neurodevelopmental outcomes at 4 to 8 years of children born at 22 to 25 weeks' gestational age: a meta-analysis. JAMA Pediatr 2013;167:967-74.

3 Costeloe KL, Hennessy EM, Haider S, et al. Short term outcomes after extreme preterm birth in England: comparison of two birth cohorts in 1995 and 2006 (the EPICure studies). BMJ 2012;345:e7976.

4 Fellman V, Hellström-Westas L, Norman M, et al. One-year survival of extremely preterm infants after active perinatal care in Sweden. JAMA 2009;301:2225-33.

5 Stoll BJ, Hansen NI, Bell EF, et al. Neonatal outcomes of extremely preterm infants from the NICHD Neonatal Research Network. Pediatrics 2010;126:443-56.

6 Itabashi K, Horiuchi T, Kusuda S, et al. Mortality rates for extremely low birth weight infants born in Japan in 2005. Pediatrics 2009;123:445-50.

7 Doyle LW, Roberts G, Anderson PJ, et al. Outcomes at age 2 years of infants $<28$ weeks' gestational age born in Victoria in 2005. J Pediatr 2010;156:49-53.

8 Rysavy MA, Li L, Bell EF, et al. Between-hospital variation in treatment and outcomes in extremely preterm infants. N Eng/ J Med 2015;372:1801-11.

9 Kollée LA, Cuttini M, Delmas D, et al. Obstetric interventions for babies born before 28 weeks of gestation in Europe: results of the MOSAIC study. BJOG 2009:116:1481-91.

10 Perlbarg J, Ancel PY, Khoshnood B, et al. Delivery room management of extremely preterm infants: the EPIPAGE-2 study. Arch Dis Child Fetal Neonatal Ed 2016;101:F384-90.

11 Marcello KR, Stefano JL, Lampron K, et al. The influence of family characteristics on perinatal decision making. Pediatrics 2011;127:e934-9.

12 Tyson JE, Parikh NA, Langer J, et al. Intensive care for extreme prematurity-moving beyond gestational age. N Engl J Med 2008;358:1672-81.

13 Berger TM. Decisions in the gray zone: evidence-based or culture-based? J Pediatr 2010;156:7-9.

14 Ancel PY, Goffinet F. EPIPAGE 2 Writing Group. EPIPAGE 2: a preterm birth cohort in France in 2011. BMC Pediatr 2014;14:97.

15 Décret no 98-900 Du 9 octobre 1998 relatif aux conditions techniques de fonctionnement auxquelles doivent satisfaire les établissements de santé pour être autorisés à pratiquer les activités d'obstétrique. de néonatologie ou de réanimation néonatale et modifiant le code de santé publique 1998.

16 Sanagou M, Wolfe R, Forbes A, et al. Hospital-level associations with 30-day patien mortality after cardiac surgery: a tutorial on the application and interpretation of marginal and multilevel logistic regression. BMC Med Res Methodol 2012;12:28.

17 Guinsburg R, Branco de Almeida MF, Dos Santos Rodrigues Sadeck L, et al. Proactive management of extreme prematurity: disagreement between obstetricians and neonatologists. J Perinatol 2012;32:913-9.

18 Serenius F, Blennow M, Maršál K, et al. Intensity of perinatal care for extremely preterm infants: outcomes at 2.5 years. Pediatrics 2015;135:e1163-72.

19 Marlow N, Bennett C, Draper ES, et al. Perinatal outcomes for extremely preterm babies in relation to place of birth in England: the EPICure 2 study. Arch Dis Child Fetal Neonatal Ed 2014;99:F181-8.

20 de Laat MW, Wiegerinck MM, Walther FJ, et al. [Practice guideline 'Perinatal management of extremely preterm delivery']. Ned Tijdschr Geneeskd 2010;154:A2701.

21 Fellman $\mathrm{V}$, Hellström-Westas L, Norman $\mathrm{M}$, et al. One-year survival of extremely preterm infants after active perinatal care in Sweden. JAMA 2009; 301:2225-33.

22 Larroque B, Bréart G, Kaminski M, et al. Survival of very preterm infants: epipage, a population based cohort study. Arch Dis Child Fetal Neonatal Ed 2004; 89:139F-44

23 Moriette G, Rameix S, Azria E, et al. [Very premature births: Dilemmas and management. Part 1. Outcome of infants born before 28 weeks of postmenstrual age and definition of a gray zone]. Arch Pediatr 2010;17:518-26.

24 Moriette $\mathrm{G}$, Rameix $\mathrm{S}$, Azria E, et al. Groupe de réflexion sur les aspects éthiques de la périnatologie. [Very premature births: Dilemmas and management. Second part: Ethical aspects and recommendations]. Arch Pediatr 2010;17:527-39.

25 Azria E, Tsatsaris V, Moriette G, et al. How to decide with precision, justice, and equity? Reflections on decision-making in the context of extreme prematurity. Part two: moving toward making the best possible decision: defining conditions for putting decisions into practice. J Gynecol Obstet Biol Reprod 2007; 36:245-52.

26 Royal College of Obstetricians \& Gynaecologists. Perinatal management of pregnant women at the threshold of infant viability (the obstetric perspective) scientific impact paper no.41. 2014 